\title{
Chloroquine blocks the autophagic process in cisplatin-resistant osteosarcoma cells by regulating the expression of p62/SQSTM1
}

\author{
CHENGCHUN SHEN $^{1 *}$, WENZHE WANG $^{2 *}$, LIJIANG TAO ${ }^{1}$, BING LIU $^{1}$, \\ ZHENGMING YANG ${ }^{1}$ and HUIMIN TAO ${ }^{1}$ \\ ${ }^{1}$ Department of Orthopedic Surgery, Second Affiliated Hospital, School of Medicine, \\ Zhejiang University, Hangzhou, Zhejiang 310009; ${ }^{2}$ Center for Molecular Medicine, \\ Zhejiang Academy of Medical Sciences, Hangzhou, Zhejiang 310013, P.R. China
}

Received February 28, 2013; Accepted May 1, 2013

DOI: $10.3892 /$ ijmm.2013.1399

\begin{abstract}
Cisplatin (DDP) is one of the most effective chemotherapeutic drugs against osteosarcoma (OS), the most common malignant bone-specific tumor. However, the acquired resistance to DDP limits its effectiveness in tumor treatment. In this study, in order to elucidate the mechanisms of drug resistance in cancer cells, we investigated cell death induced by DDP in OS cells. We evaluated the contribution of autophagy in the process of drug resistance in a panel of four OS cell lines, MG-63, U-2OS, MNNG/HOS and Saos-2. The cells were treated with DDP $(0-50 \mu \mathrm{M})$ for $48 \mathrm{~h}$ and then cell vaibility was assessed using the Cell Counting kit-8 (CCK-8). Apoptosis was detected by flow cytometry and the green fluorescent protein (GFP)-microtubule-associated protein 1 light chain 3 (LC3) expression vector was used to visualize the formation of autophagic vesicles. Our results demonstrated that autophagy was induced by DDP in the drug-resistant cell line, Saos-2, which does not respond to DDP with apoptosis. DDP-induced autophagy protected the Saos- 2 cells from apoptotic cell death. Moreover, the inhibition of autophagy by chloroquine, an inhibitor of lysosomal proteases, accelerated the DDP-induced cell death in Saos- 2 cells. We also found that during DDP treatment, the protein expression level of the autophagic regulator, p62/sequestosome 1 (SQSTM1), decreased during the first hour of treatment, followed by a rapid recovery. Therefore, our data suggest a potential clinical therapy by targeting autophagy with chloroquine or monoclonal antibodies for the treatment of drug-resistant OS.
\end{abstract}

Correspondence to: Professor Huimin Tao, Department of Orthopedic Surgery, Second Affiliated Hospital, School of Medicine, Zhejiang University, 88 Jie Fang Road, Hangzhou, Zhejiang 310009, P.R. China

E-mail: huimintao_zrgk@163.com

${ }^{*}$ Contributed equally

Key words: osteosarcoma, autophagy, cisplatin, chloroquine, p62/sequestosome 1

\section{Introduction}

Osteosarcoma (OS) is the most common specific malignant bone tumor. The five-year survival rate of patients with OS has increased to $60 \%$ with the current combination of modern surgery and systemic chemotherapy (1). Cisplatin (DDP), which functions in a manner similar to alkylating agents, is one of the most effective drugs against OS. However, acquired resistance to DDP by tumor cells significantly limits its efficacy for the treatment of OS. Such resistance is closely associated with the recurrence and metastasis of OS following traditional treatment. The failure of DDP to induce apoptosis programmed cell death (PCD) type I is considered one of the major mechanisms underlying resistance to DDP $(2,3)$. Effective systemic therapeutic options are therefore required to eliminate the primary lesion, particularly in drug-resistant OS.

Autophagy is a catabolic process whereby cells maintain homeostasis by eliminating unnecessary proteins and damaged organelles $(4,5)$. Autophagy is associated with a number of physiological processes, including development, differentiation, neurodegeneration, infection and cancer (6). Previous studies have demonstrated that autophagy induced in cancer cells by anticancer drugs may contribute to cancer cell survival in growth-limiting conditions, such as nutrient depletion, hypoxia, absence of growth factors and anticancer drug treatments (7-12). Furthermore, autophagy protects cancer cells from anticancer drug-induced apoptosis and promotes their survival and recovery following treatment with chemotherapeutic drugs $(13,14)$.

p62/sequestosome 1 (SQSTM1) is an ubiquitin-binding protein involved in cell signaling, oxidative stress and autophagy (15-19). Considering that the induction of autophagy is accompanied by $\mathrm{p} 62 / \mathrm{SQSTM} 1$ protein degradation, (autophagy usually downregulates the p62 protein level by degradation, not DNA transcription/tranlation), determining the intracellular level of p62/SQSTM1 by western blot analysis is used routinely to measure the autophagic flux in response to pro-autophagic stimuli. It has been reported that silencing the expression of the microtubule-associated protein 1 light chain 3 (LC3) protein triggers the intracellular accumulation of ubiquitinated protein aggregates bound to p62/SQSTM1 and/or neighbor of BRCA1 gene 1 (NBR1) protein $(18,19)$. Linares et al showed that the CDK1-dependent phosphorylation of p62/SQSTM1 places a 
restraint on tumor transformation, highlighting another function of p62/SQSTM1, which can act as an oncogene depending on the cellular circumstances (20).

In this study, we demonstrate that autophagy is induced by DDP in the Saos-2 cell line, an OS cell that does not respond to DDP with apoptosis. The induced autophagy protected the Saos-2 cells from apoptotic cell death induced by DDP. Moreover, the inhibition of autophagy by chloroquine, an autophagic inhibitor that blocks lysosomal degradation, accelerated the DDP-induced cell death of Saos-2 cells. Thus, autophagy appears to protect drug-resistant OS cells from DDP-induced apoptosis. Moreover, our data indicate that chloroquine suppresses the autophagic process in DDP-resistant OS cells by regulating the expression of p62/SQSTM1, which plays a critical role in the progression and the drug resistance of OS.

\section{Materials and methods}

Cell culture. The established human OS cell lines, MG-63 (CRL-1427 ${ }^{\mathrm{TM}}$; ATCC, Manassas, VA, USA), U-2OS (HTB-96 ${ }^{\mathrm{TM}}$; ATCC), MNNG/HOS (CRL-1547 ${ }^{\mathrm{TM}}$; ATCC) and Saos-2 (HTB-85; ATCC), were obtained from the Cell Bank of the Shanghai Institute of Biochemistry and Cell Biology, Chinese Academy of Sciences (Shanghai, China), where they were tested and authenticated. All cell lines were grown in culture medium supplemented with $1 \%$ penicillin/streptomycin and 10\% (MG-63, $\mathrm{U}-2 \mathrm{OS}$ and MNNG/HOS) or $15 \%(\mathrm{Saos}-2)(\mathrm{v} / \mathrm{v})$ fetal bovine serum (Gibco, Grand Island, NY, USA) at $37^{\circ} \mathrm{C}$ in $5 \% \mathrm{CO}_{2}$.

Reagents and antibodies. DDP (P4394), E-64d (E8640), chloroquine (C6628) and pepstatin A (P5318) were obtained from Sigma-Aldrich (St. Louis, MO, USA). The anti-LC3 and anti-p62/SQSTM1 antibodies were purchased from Sigma-Aldrich, and the antibodies directed against caspase-3, autophagy protein 5 (ATG5), beclin 1, Barkor/ATG14 and phosphatidylinositol-3-kinase (PI3K) C3 were purchased from Cell Signaling Technology (Danvers, MA, USA).

Cell Counting kit-8 (CCK-8) viability assay. The cells were seeded at $8 \times 10^{3}$ (MG-63, HOS and U-2OS) and $1 \times 10^{4}$ (Saos-2) cells/well in 96-well plates, with concentrations of 0-50 $\mu \mathrm{M}$ DDP for $48 \mathrm{~h}$ and incubated for an additional $60 \mathrm{~min}$ at $37^{\circ} \mathrm{C}$ in $10 \% \mathrm{CCK}-8$ dye (Dojindo, CK04). Water-soluble tetrazolium salt (WST-8) is reduced by the dehydrogenases in the cells to create an orange-colored product (formazan) that is soluble in the cell culture medium. The amount of the formazan dye generated by the dehydrogenases in the cells is directly proportional to the number of living cells and is determined by the absorbance at $450 \mathrm{~nm}$.

Flow cytometry: quantification of apoptosis. To evaluate the level of apoptosis, the cells floating in the medium and the adherent cells were collected after $48 \mathrm{~h}$ of treatment. The cells were stained using the Annexin V-FITC Apoptosis Detection kit (Sigma-Aldrich) according to the manufacturer's instructions. The samples were analyzed using a FACSCalibur flow cytometer (Becton-Dickinson, North Ryde, New South Wales, Australia).

Electron microscopy. Untreated and treated cells were fixed in $2.5 \%$ glutaraldehyde at room temperature for $40 \mathrm{~min}$. Cells were post-fixed in $1 \%$ osmium tetroxide at room temperature for $60 \mathrm{~min}$, dehydrated in a series of increasing concentrations of ethanol solutions (50, 70, 95 and 100\%), and embedded in epoxy resin prior to sectioning. Representative regions were selected for ultra-thin sectioning and the samples were examined under an electron microscope.

Green fluorescent protein (GFP)-LC3 transfection. The GFP-LC3 expression vector was used to visualize the formation of autophagic vesicles. Cells at $90 \%$ confluency were transiently transfected with GFP-LC3 using Lipofectamine 2000 (Invitrogen, Carlsbad, CA, USA) according to the manufacturer's instructions. To obtain the highest transfection efficiency and lowest cytotoxicity, we optimized the ratio of the DNA to Lipofectamine 2000. Twenty-four hours post-transfection, the cells were treated with different reagents, fixed with $4 \%$ paraformaldehyde in PBS and placed on slides. The assessment of GFP fluorescence was carried out using a fluorescence microscope (Nikon, Tokyo, Japan).

Western blot analysis. The cells were seeded in cell culture flasks or 6-well plates and cultured until they reached approximately $80 \%$ confluency. Fresh medium was added before further processing. Total cellular protein extracts were prepared by scraping the cells into Mammalian Protein Extraction Reagent (78503; Thermo Scientific, Waltham, MA, USA). The protein concentration was measured using a BCA protein assay (Thermo Scientific Pierce). The proteins were separated by SDS-PAGE $10-15 \%$ and electrotransferred onto either nitrocellulose or polyvinylidene fluoride (PVDF) membranes (Millipore, Billerica, MA, USA). The membranes were blocked with $5 \%$ bovine serum albumin (BSA) in Tris-buffered saline containing 0.1\% Tween-20 (TBST, pH 7.6) for $90 \mathrm{~min}$ at room temperature. All primary antibodies were incubated overnight at $4^{\circ} \mathrm{C}$. The membranes were incubated in secondary antibodies diluted in 5\% BSA in TBST for $90 \mathrm{~min}$ at room temperature with mild agitation. The membranes were washed three times between each of the incubations. The labeled proteins were detected by enhanced chemiluminescence (ECL kit; Bio-Rad, Hercules, CA, USA).

Statistical analysis. The data are expressed as the means \pm SD. The statistical significance of the differences was determined using a Student's two-tailed t-test for two groups and one-way ANOVA for multiple groups. P-values $<0.05$ were considered to indicate statistically significant differences. All data were analyzed using SPSS 16.0 software.

\section{Results}

DDP-induced cell death in OS cells. We evaluated a group of four OS cell lines, MG-63, U-2OS, MNNG/HOS and Saos-2, for their sensitivity to the chemotherapeutic drug, DDP. DDP $(10 \mu \mathrm{M})$ inhibited cell proliferation in the U-2OS and MNNG/HOS cells. The MG-63 cells were marginally more resistant to DDP treatment compared to the U-2OS and MNNG/ HOS cells, and the Saos-2 cells were completely resistant to DDP treatment at $10 \mu \mathrm{M}$ or even higher concentrations (Fig. 1A).

We then examined the typical markers of apoptotic cell death in the four cell lines. Th expression of cleaved caspase- 3 
A

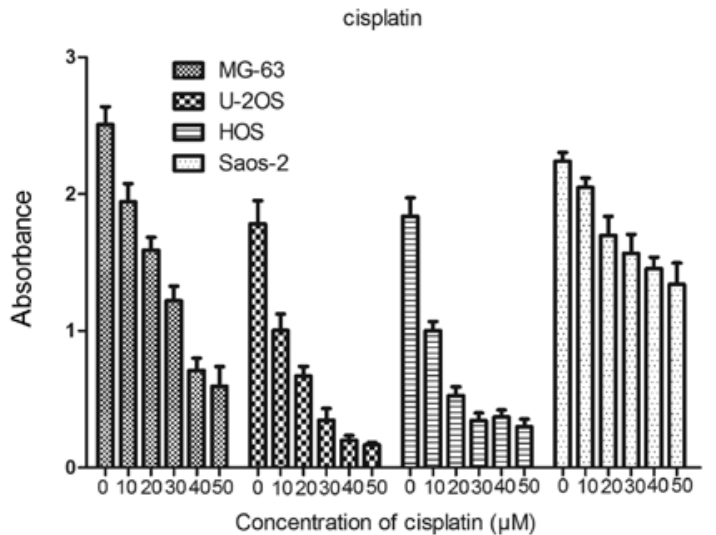

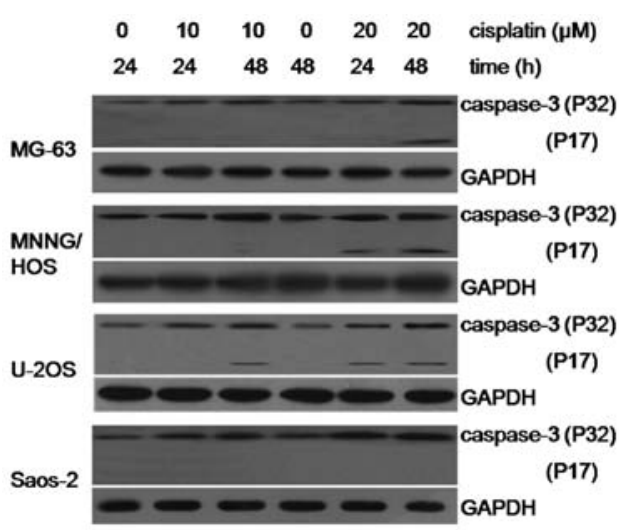

C

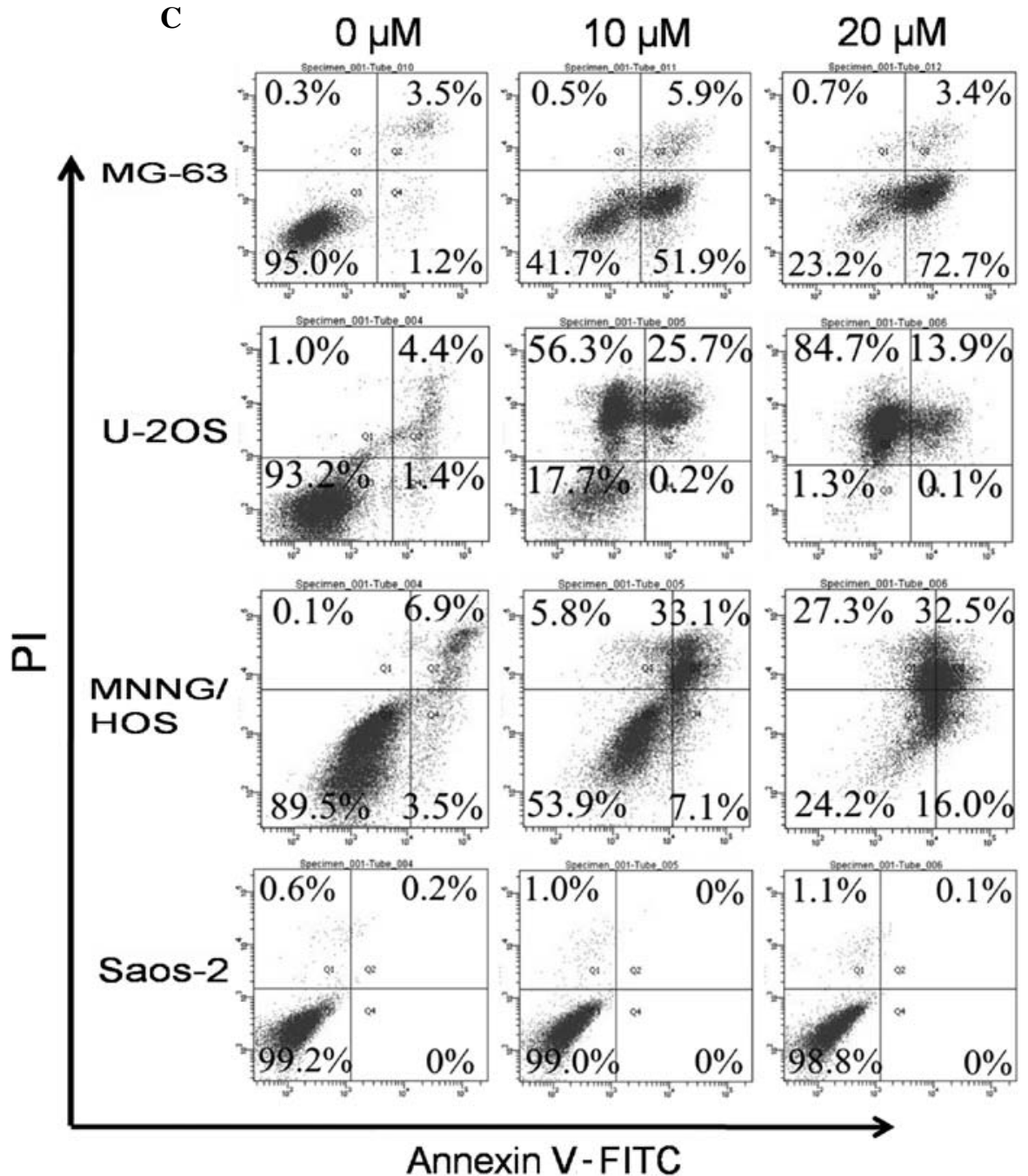

Figure 1. Analysis of cisplatin (DDP)-induced osteosarcoma (OS) cell death. (A) MG-63, HOS and U-2OS cells seeded at 8x10 3 cells/well and Saos-2 cells seeded at $1 \times 10^{4}$ cells/well in a 96-well plate (Saos-2 cells grow at a slower rate and were therefore seeded at a higher density than the other cells) were treated with various concentrations (10-50 $\mu \mathrm{M}$ ) of DDP for $48 \mathrm{~h}$. The Cell Counting kit-8 (CCK-8( assay was used to determine the sensitivity of each cell line to DDP. (B) Cells were treated with 0,10 or $20 \mu \mathrm{M}$ DDP for 24 or $48 \mathrm{~h}$ and then subjected to western blot analysis using an anti-caspase-3 antibody. GAPDH served as the loading control. (C) Annexin-V/PI apoptosis assay of OS cells treated with 0,10 or $20 \mu \mathrm{M}$ DDP for $48 \mathrm{~h}$. The percentages of live (lower-left quadrant), early apoptotic (lower-right quadrant), late apoptotic (upper-right quadrant) and necrotic (upper-left quadrant) cells are shown.

was assessed following treatment with DDP (Fig. 1B). The expression of cleaved caspase-3 was significantly increased in the U-2OS, MNNG/HOS and MG-63 cells, whereas no induction of cleaved caspase- 3 expression was observed in the Saos-2 cells. To quantitatively evaluate apoptosis in the four cell lines, we exposed the cells to 0,10 or $20 \mu \mathrm{M}$ DDP for $48 \mathrm{~h}$, and the apoptosis rate was determined using the Annexin V-PI (AV-PI) assay. Fig. $1 \mathrm{C}$ shows the flow cytometric analyses representative of three independent experiments. Both the drug-sensitive (U-2OS and MNNG/HOS) cells and the relatively resistant MG-63 cells demonstrated necrosis and apoptosis (76.1\% apoptosis rate and $0.7 \%$ necrosis rate for MG-63, $14 \%$ apop- 
A

MG-63

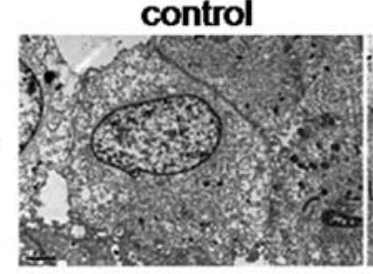

cisplatin $(40 \mu \mathrm{M})$

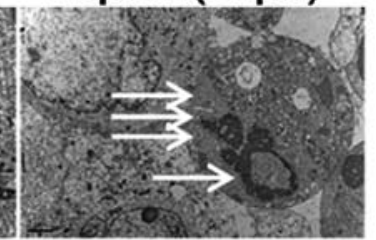

U-2OS

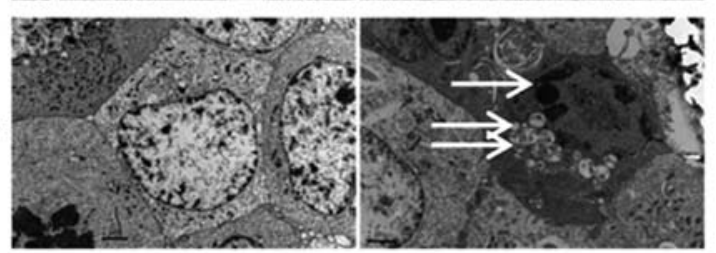

MNNG/ HOS
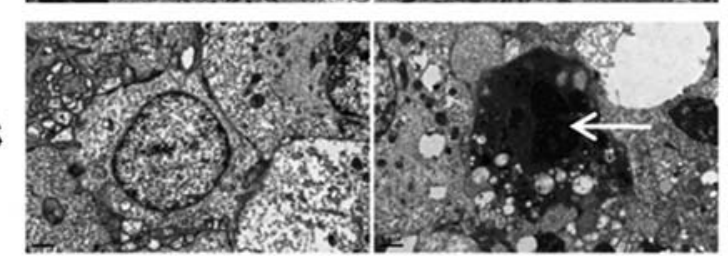

Saos-2

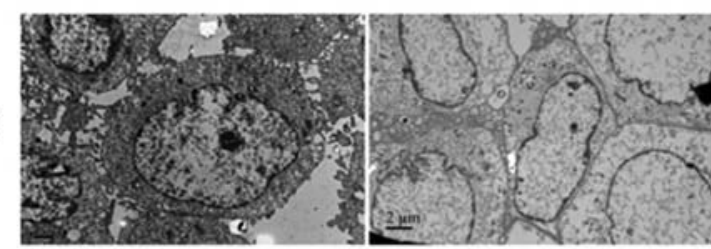

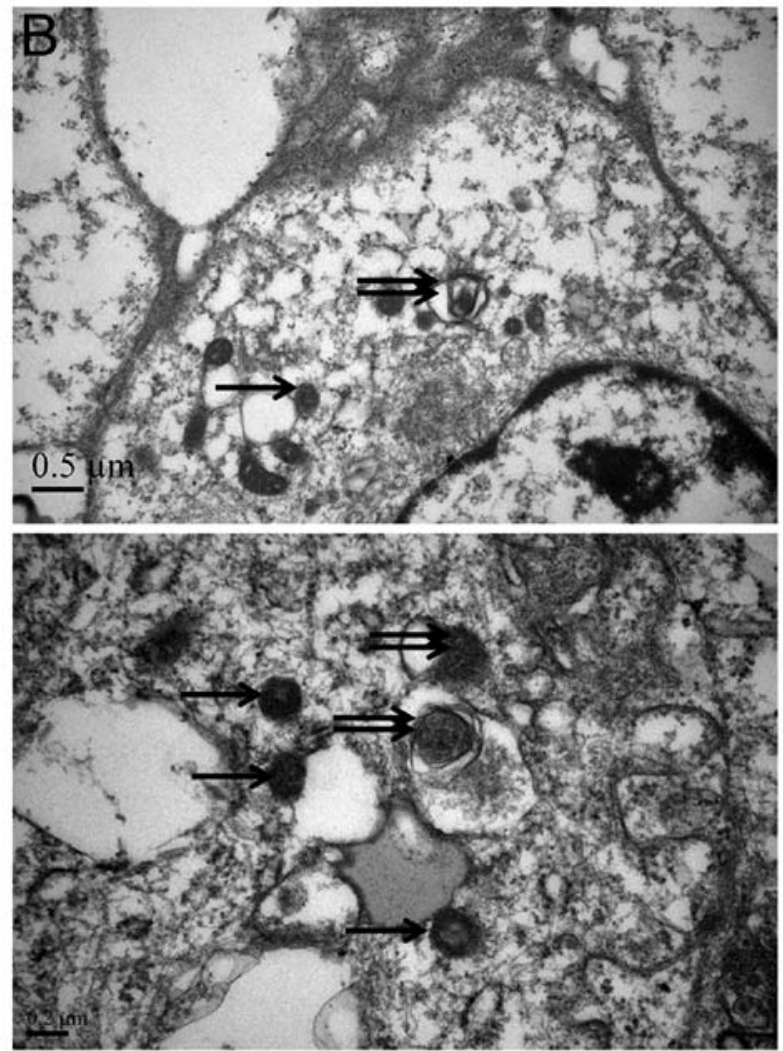

Figure 2. Examination of osteosarcoma (OS) cells under an electron microscope. (A) Representative electron microscopic images of MG-63, HOS, U-2OS and Saos-2 cells untreated (control) or treated with cisplatin (DDP) $(40 \mu \mathrm{M})$ for $48 \mathrm{~h}$. The apoptotic features include cell shrinkage, chromatin marginalization (white arrows) and an intact but blebbed plasma membrane (double white arrows); whereas the late apoptotic phenomena are nuclear debris and apoptotic bodies (triplicate white arrows). Non-apoptotic programmed cell death (PCD) type II features include the apparent disintegration of numerous cytoplasmic vacuoles. (B) Black arrows indicate the nascent autophagosomes. For each treatment or control group, transmission electron microscope images were randomly chosen from a field of at least 100 cells.

tosis rate and $84.7 \%$ necrosis rate for $\mathrm{U}-2 \mathrm{OS}, 48.5 \%$ apoptosis rate and $27.3 \%$ necrosis rate for $\mathrm{MNNG} / \mathrm{HOS}$ ) in response to DDP $(20 \mu \mathrm{M})$. By contrast, the significantly drug-resistant (Saos-2) cells had a very low rate of apoptosis $(0.1 \%)$.

DDP induces authentic autophagy in the Saos-2 cells Comparison of the morphological features of four DDP-treated OS cells observed under an electron microscope. The U-2OS, MG-63 and MNNG/HOS cells treated with DDP (40 $\mu \mathrm{M})$ for $48 \mathrm{~h}$ showed typical apoptotic morphology, including cell shrinkage and chromatin marginalization with an intact but blebbed plasma membrane and late apoptotic phenomena, such as nuclear debris and apoptotic body formation (Fig. 2A). By contrast, the DDP-treated Saos-2 cells retained an intact nuclear membrane containing a distinct nucleolus, with areas of more electron-dense heterochromatin. In addition, higher magnification images revealed the presence of numerous cytoplasmic vacuoles resembling nascent autophagosomes, many of which appeared to surround cytoplasmic material and components, such as the mitochondria (Fig. 2B).

DDP induces the formation and degradation of autophagosomes in Saos-2 cells. LC3, an autophagosomal membrane protein, is widely used as a cellular marker to assess the quantity of autophagosomes in cells (21). LC3 with an N-terminus GFP has been used to monitor autophagy through direct fluo- rescence microscopy. Diffuse GFP fluorescence was observed in the U-2OS, MG-63 and MNNG/HOS and Saos-2 cells expressing GFP-tagged LC3 using a laser scanning confocal fluorescence microscope. Moreover, the number of GFP-LC3 puncta in the Saos-2 cells significantly increased following exposure to DDP (48 h) (Fig. 3A). These results suggest that DDP induces autophagy in the Saos-2 cells.

The formation of a lipidation form of LC3, termed LC3-II is induced by autophagy. Thus, it is a reliable biochemical marker for autophagy. We therefore investigated the conversion of LC3 form I (LC3-I, $18 \mathrm{kDa}$ ) to form II (LC3-II, $16 \mathrm{kDa}$ ) by western blot analysis. The results revealed that the U-2OS, MG-63 and MNNG/HOS cells did not convert LC3-I to LC3-II following exposure to DDP. By contrast, the levels of LC3-I and LC3-II were downregulated in the Saos-2 cells that were treated with DDP for $48 \mathrm{~h}$ (Fig. 3B). This is may be due to the rapid lysosomal degradation of LC3-II during autophagy. Thus, we believe that DDP indeed induces autophagy in the Saos-2 cells.

To elucidate the molecular mechanism by which DDP induces autophagy in the Saos- 2 cells, we co-treated the cells with different autophagic inhibitors. As shown in Fig. $3 \mathrm{Ci}$, GFP-LC3 puncta were not observed when the cells were treated with the lysosomal protease inhibitor, chloroquine (6 $\mu \mathrm{M}$, added $2 \mathrm{~h}$ prior to exposure to DDP); however, the degradation of autophagosomes was not blocked by the other inhibitors of lysosomal proteases, E64d and pepstatin A. The 


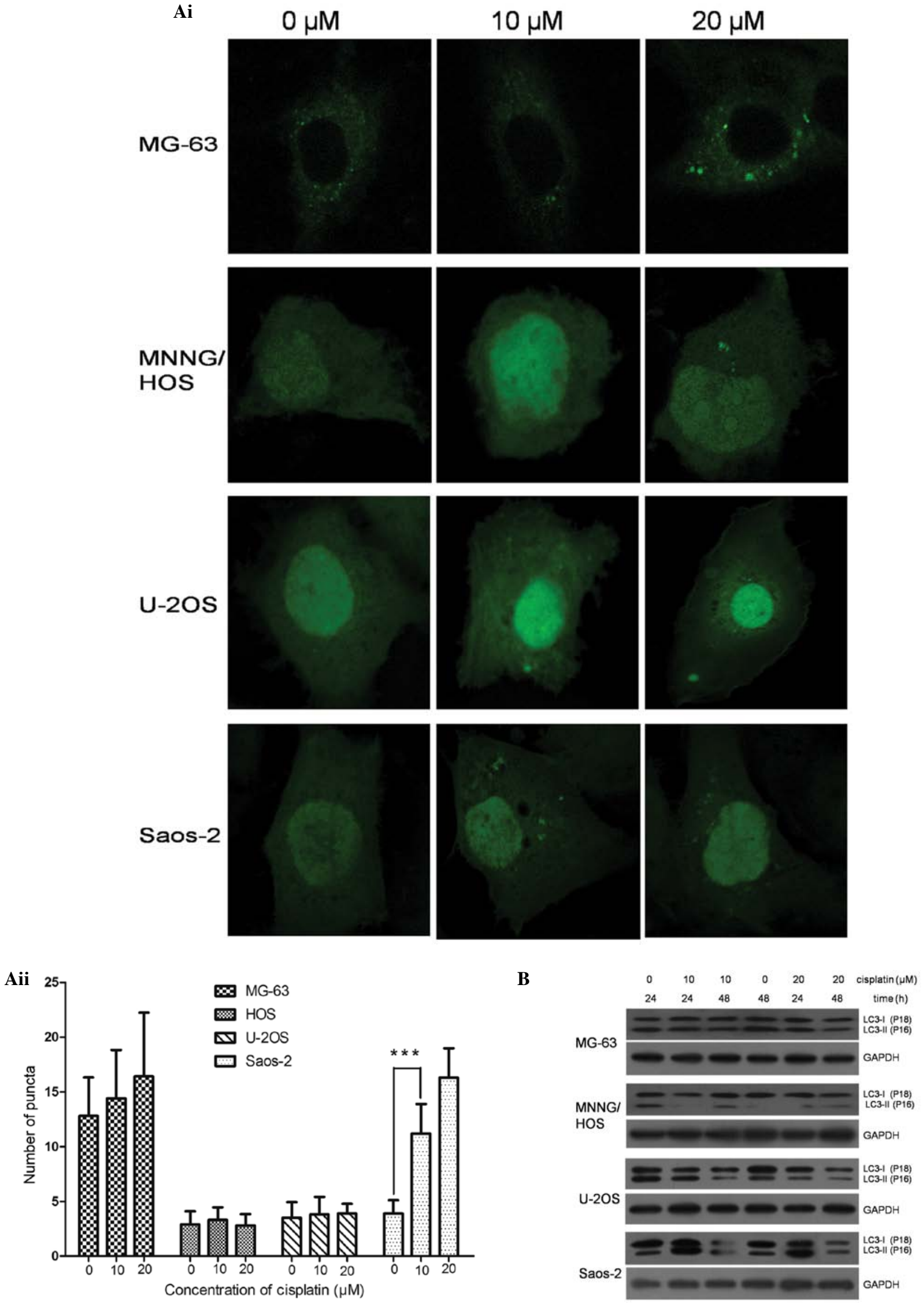

Figure 3. Autophagic features induced by cisplatin (DDP). (A) Fluorescent microscopic images (magnification x63) of green fluorescent protein (GFP)microtubule-associated protein 1 light chain 3 (LC3)-transfected cells treated with 0,10 or $20 \mu \mathrm{M}$ of DDP for $48 \mathrm{~h}$. MG-63, MNNG/HOS, U-2OS and Saos-2 cells display GFP-LC3 diffusely distributed throughout the cytoplasm (i) and puncta (ii). Mean \pm SEM, $\mathrm{n}=10$. (B) Cells were treated with 0,10 or $20 \mu \mathrm{M}$ of DDP for 24 or $48 \mathrm{~h}$ and then subjected to western blot analysis using anti-LC3 antibody. GAPDH served as the loading control. 

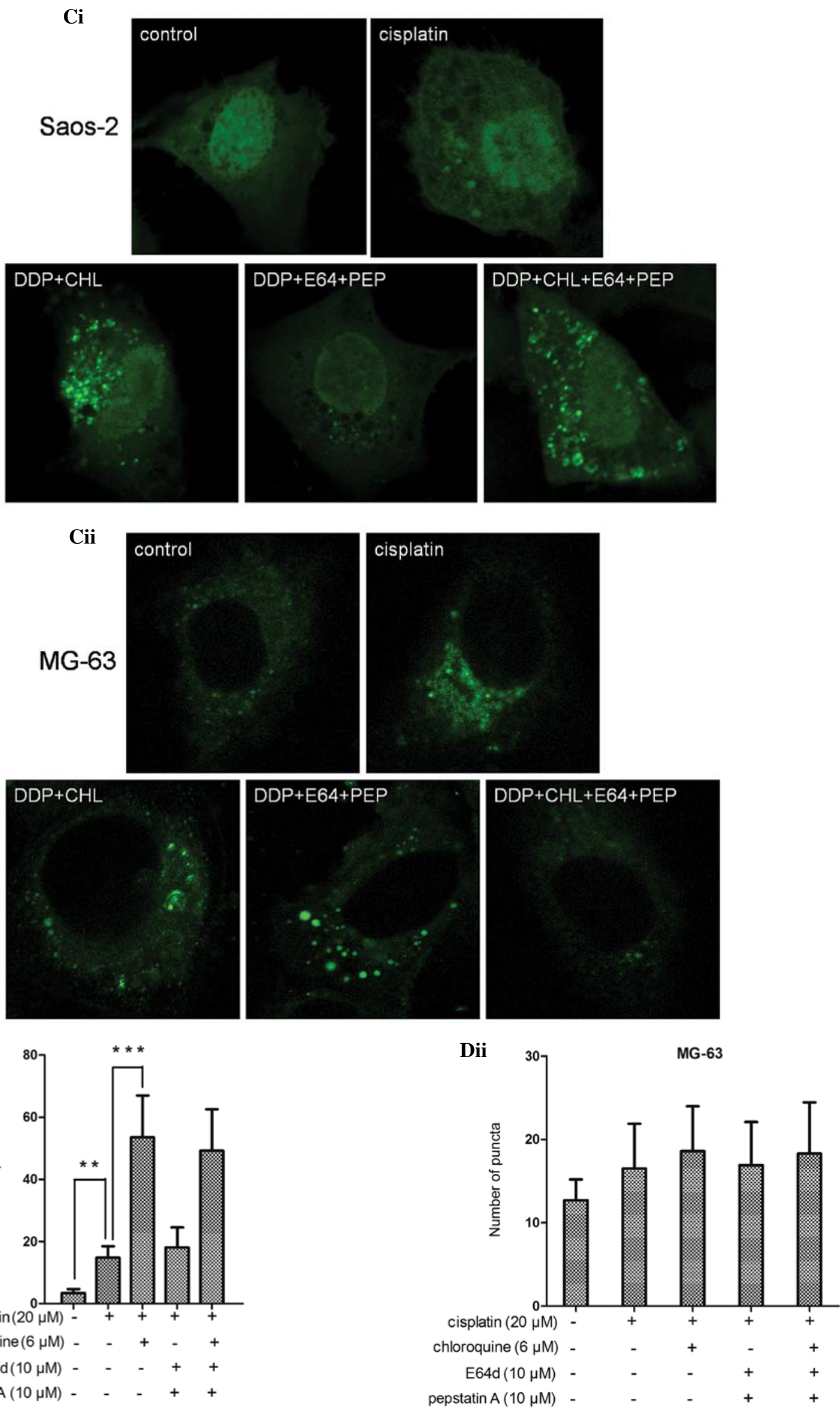

Figure 3. Continued. (Ci) Saos-2 and (Cii) MG-63 cells were transfected with green fluorescent protein (GFP)-tagged microtubule-associated protein 1 light chain 3 (LC3); $24 \mathrm{~h}$ after transfection, the cells were treated with $20 \mu \mathrm{M}$ cisplatin (DDP) in the presence or absence of chloroquine $(6 \mu \mathrm{M})$ for $48 \mathrm{~h}$ or with E64d $(10 \mu \mathrm{M})$ and pepstatin A $(10 \mu \mathrm{M})$ for $48 \mathrm{~h}$. (D) The number of GFP-LC3-positive puncta in individual (i) Saos-2 and (ii) MG-63 cells was counted in at least 100 cells for each different treatment group. The results are shown as the means \pm SD. 


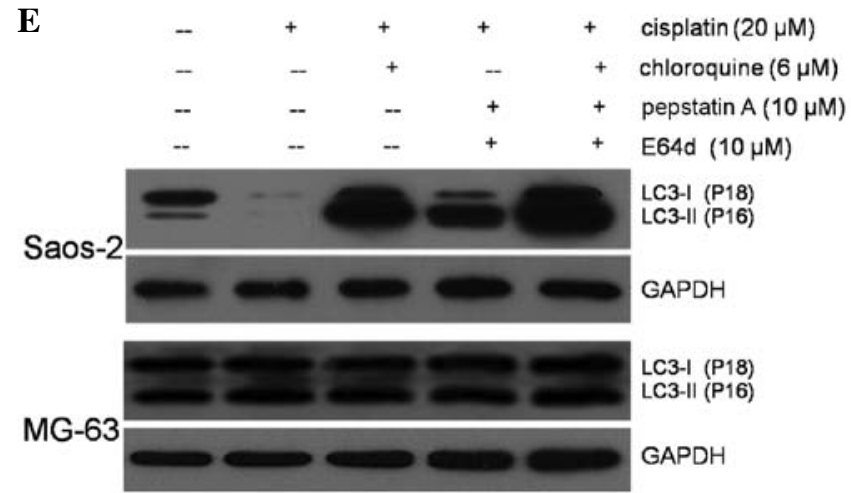

Figure 3. Continued. (E) Total lysates of the cells were analyzed by immunoblot analysis using anti-LC3 antibody. GAPDH served as the loading control. $\left({ }^{* * *} \mathrm{P}<0.001\right)$. LC3, microtubule-associated protein 1 light chain 3.

average number of GFP-LC3-positive puncta in ten typical (x63 magnifications) cells was counted to measure the statistical differences. Histograms revealed that only chloroquine affected the degradation of the autophagosomes (Fig. 3Di). This is consistent with the fluorescence microscopy data. The results from western blot analysis demonstrated that LC3-II was significantly upregulated in the Saos-2 cells treated with a combination of DDP and chloroquine, compared to the Saos-2 cells treated with DDP alone (Fig. 3E). However, the accumulation of LC3-II and the inhibition of lysosomal proteases was not observed in the MG-63 cells, which are mildly resistant to DDP-induced apoptosis (Fig. 3Cii and Dii).

Taken together, these results provided strong support to the conclusion that while DDP induces apoptosis in the U-2OS, MG-63, MNNG/HOS cells it causes autophagy in the Saos-2 cells. Furthermore, autophagy protected the Saos- 2 cells from DDP-induced cell death.

Chloroquine blocks the DDP-induced autophagy in Saos-2 cells by regulating p62/SQSTM1. The protein levels of autophagic substrates can be used to monitor the autophagic flux. A previous study revealed that several specific substrates are preferentially degraded by autophagy, among which the most extensively studied autophagic substrate, is p62/SQSTM1 (22). In this study, we examined the expression of p62/SQSTM1 in the Saos-2 and MG-63 cells treated with 0, 10 and $20 \mu \mathrm{M}$ DDP for $48 \mathrm{~h}$. The downregulation of p62/SQSTM1 was observed in the Saos-2 cells $48 \mathrm{~h}$ following exposure to DDP (Fig. 4A). To examine such a regulation in further detail, we examined the expression of $\mathrm{p} 62 / \mathrm{SQSTM} 1$ at various time points over a period of $24 \mathrm{~h}$ in the Saos-2 and MG-63 cells treated with $20 \mu \mathrm{M}$ DDP. In the Saos-2 cells, the expression level of p62/ SQSTM1 decreased after $1 \mathrm{~h}$ of treatment with DDP, followed by a gradual recovery (Fig. 4B). These results suggest that the total cellular expression levels of p62/SQSTM1 correlate with the autophagic activity and that p62/SQSTM1 plays a potential role in the response to DDP in the Saos-2 cells. A previous study revealed that the inhibition of autophagy in HeLa cells resulted in the accumulation of p62/SQSTM1 (22). As shown in Fig. 4C, the downregulation of p62/SQSTM1 expression was observed when autophagy was inhibited by the lysosomal protease inhibitor, chloroquine ( $6 \mu \mathrm{M}$, added $2 \mathrm{~h}$ prior to exposure to DDP);
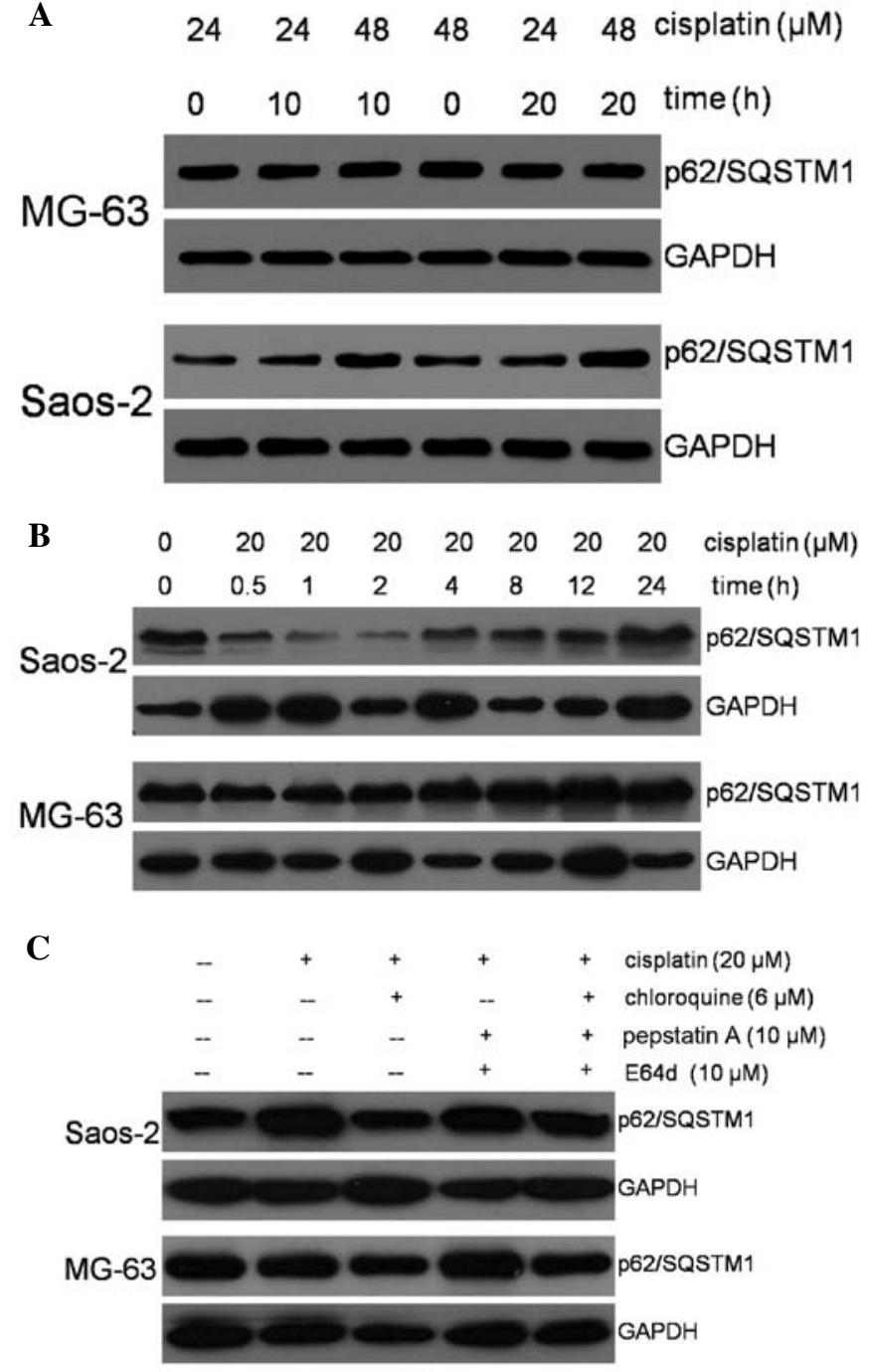

D

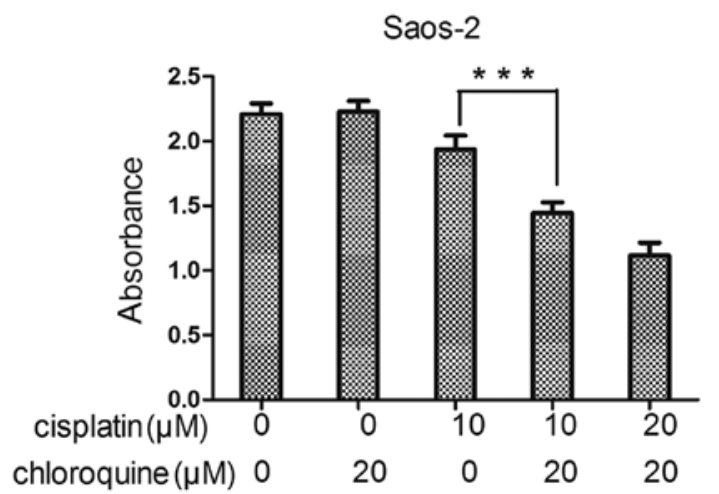

Figure 4. Regulation of p62/SQSTM1 protein expression in osteosarcoma (OS) cells under cisplatin (DDP)-induced autophagic conditions. (A) Saos-2 and MG-63 cells were treated with 0,10 or $20 \mu \mathrm{M}$ DDP for 24 or $48 \mathrm{~h}$ and then subjected to western blot analysis using anti-p62/SQSTM1 antibody. GAPDH served as the loading control. (B) Saos-2 and MG-63 cells were treated with $20 \mu \mathrm{M}$ DDP for the indicated times, and subjected to immunoblot analysis using anti-p62/SQSTM1 antibody. GAPDH served as the loading control. Downregulation of p62/SQSTM1 was detected $1 \mathrm{~h}$ after treatment with DDP. Furthermore, the normal expression of p62/SQSTM1 was regained within 24 h. (C) Saos-2 and MG-63 cells were treated with $20 \mu \mathrm{M}$ DDP in the presence or absence of chloroquine $(6 \mu \mathrm{M})$ for $48 \mathrm{~h}$ or with E64d $(10 \mu \mathrm{M})$ and pepstatin $\mathrm{A}(10 \mu \mathrm{M})$ for $48 \mathrm{~h}$, and then subjected to immunoblot analysis using anti-p62/SQSTM1 antibody. GAPDH served as the loading control. (D) Saos- 2 cells seeded at $1 \times 10^{4}$ cells/well of a 96-well plate were treated with various concentrations $(0-20 \mu \mathrm{M})$ of DDP and/or $20 \mu \mathrm{M}$ of chloroquine for $48 \mathrm{~h}$. The CCK- 8 assay was used to determine the sensitivity of Saos-2 cells to DDP and/or chloroquine $\left({ }^{* * *} \mathrm{P}<0.001\right)$. 
however, the formation of autophagosomes was not blocked by E64d or pepstatin A, two other inhibitors of lysosomal proteases. Accordingly, we found that DDP in combination with chloroquine inhibited cell proliferation in a dose-dependent manner (Fig. 4D). These data suggest that chloroquine inhibits the autophagic process in DDP-resistant OS cells by regulating the expression or degradation of p62/SQSTM1.

\section{Discussion}

In recent decades, although the survival rate of patients with OS has increased as a result of rapid advancements in comprehensive therapy, particularly neoadjuvant chemotherapy, the cytotoxic effects of drugs on OS cells are reduced by acquired chemoresistance. Therefore, the specific mechanisms of drug resistance and the molecular targets involved must be explored to overcome the resistance to cytotoxic drugs. Autophagy may help cancer cells survive in growth-limiting conditions, such as the presence of anticancer drugs. In this study, we demonstrate that DDP-sensitive cells underwent authentic apoptosis, with the cells exhibiting caspase-dependent death. Of note, only a small number of Saos-2 cells underwent apoptosis following treatment with DDP. Furthermore, the results revealed that DDP induced autophagy in a dose- and time-dependent manner in this DDP-resistant cell line, which may be the survival factor facilitating their development of acquired resistance.

Autophagy is a catabolic process for the autophagosomiclysosomal degradation of bulk cytoplasmic content. The autophagic flux can be measured by measuring the LC3-II turnover in the presence or absence of lysosomal degradation, which can be assessed by western blot analysis (20). Lysosomal degradation can be prevented by certain protease inhibitors (e.g., chloroquine, pepstatin A and E64d) (23). In our study, despite the salient attenuation of LC3-I, the conversion of endogenous LC3-I to LC3-II in the DDP-treated Saos-2 cells was insignificant. Therefore, we examined the turnover of LC3-II in the Saos-2 cells treated with DDP in combination with different lysosomal inhibitors (chloroquine or pepstatin A and E64d) by western blot analysis and laser confocal fluorescence microscopy. The results revealed that the DDP-induced aggregation of LC3-II was significantly reduced in the Saos-2 cells treated with chloroquine and DDP but not when they were treated with chloroquine plus pepstatin A and E64d. These results were confirmed by the fluorescent data, which demonstrated the accumulation of GFP-LC3 puncta in the Saos-2 cells treated with DDP in combination with lysosomal protease inhibitors. In addition, the levels of other autophagic substrates may be used to monitor the autophagic flux. The adaptor protein, p62/SQSTM1, is required for the formation of ubiquitinated protein aggregates in vitro. p62/SQSTM1 is selectively incorporated into autophagosomes through its direct binding with LC3. Therefore, the total cellular expression levels of p62/SQSTM1 inversely correlate with autophagic activity. In our study, our results revealed that the expression level of p62/SQSTM1 was upregulated following treatment with DDP for $48 \mathrm{~h}$. These results suggest that the analysis of p62/SQSTM1 expression may be useful in assessing the early induction of autophagy and may also be a marker of autophagy. More importantly, p62/SQSTM1 plays a significant role in the resistance of OS cells to DDP.
Chloroquine has been used for the treatment of various diseases, such as malaria and rheumatoid arthritis for several decades (23). The application of chloroquine as an adjuvant anticancer drug has previously been reported as it plays a key role in halting the DNA repair process. In addition, treatment with chloroquine leads to an apparent increase in the formation of autophagosomes, possibly by blocking the fusion of lysosomes (24). For this reason, in this study, we investigated the potential cytotoxic effects of three lysosomal protease inhibitors (chloroquine, E64d and pepstatin A). Furthermore, we assessed the contribution of DDP-induced autophagy to the survival of Saos-2 cells, using the lysosomotropic agent chloroquine, which prevents lysosomal degradation. The marked increase in cell death observed following co-treatment with DDP and chloroquine suggested that the addition of a late phase autophagic inhibitor to DDP reduced the chemoresistance of the Saos- 2 cells. Notably, the addition of chloroquine diminished the DDP-induced downregulation of p62/SQSTM1 expression in the Saos-2 cells, suggesting that chloroquine blocked the DDP-induced autophagy in the drug-resistant OS cells by regulating the expression of p62/SQSTM1 and LC3. The combination of an autophagic inhibitor with agents that induce apoptosis in drug-resistant cancer cells as a survival response has recently been proposed as a novel targeted therapy for cancer $(25,26)$. Thus, chloroquine appears to be a promising candidate for the inhibition of autophagy in OS cells, which has long been used as an anti-malarial and antirheumatic drug.

In conclusion, in this study, we demonstrate that autophagy is a protective mechanism induced in DDP-resistant human OS cells in vitro. In addition, chloroquine dramatically suppressed the autophagic process that led to apoptotic cell death in the DDP-resistant OS cells by regulating the expression of LC3 and p62/SQSTM1. Our data suggest a potential clinical therapy targeting autophagy with chloroquine or monoclonal antibodies for the treatment of drug-resistant OS.

\section{Acknowledgements}

This study was supported in part by a grant (2012C24008) from the Science Technology Department of Zhejiang Province. We thank Qing Zhong (University of California at Berkeley) for providing the GFP-LC3 plasmid.

\section{References}

1. Bacci G, Ferrari S, Bertoni F, et al: Long-term outcome for patients with nonmetastatic osteosarcoma of the extremity treated at the istituto ortopedico rizzoli according to the istituto ortopedico rizzoli/osteosarcoma-2 protocol: an updated report. J Clin Oncol 18: 4016-4027, 2000.

2. Brozovic A and Osmak M: Activation of mitogen-activated protein kinases by cisplatin and their role in cisplatin-resistance. Cancer Lett 251: 1-16, 2007.

3. Gorlick R, Anderson P, Andrulis I, et al: Biology of childhood osteogenic sarcoma and potential targets for therapeutic development: meeting summary. Clin Cancer Res 9: 5442-5453, 2003.

4. Reggiori F and Klionsky DJ: Autophagy in the eukaryotic cell. Eukaryot Cell 1: 11-21, 2002

5. Codogno P and Meijer AJ: Autophagy and signaling: their role in cell survival and cell death. Cell Death Differ 12 (Suppl 2): 1509-1518, 2005.

6. Levine B and Yuan J: Autophagy in cell death: an innocent convict? J Clin Invest 115: 2679-2688, 2005. 
7. Kondo Y, Kanzawa T, Sawaya R and Kondo S: The role of autophagy in cancer development and response to therapy. Nat Rev Cancer 5: 726-734, 2005.

8. Krysko DV and Vandenabeele P: From regulation of dying cell engulfment to development of anti-cancer therapy. Cell Death Differ 15: 29-38, 2008.

9. Degenhardt K, Mathew R, Beaudoin B, et al: Autophagy promotes tumor cell survival and restricts necrosis, inflammation, and tumorigenesis. Cancer Cell 10: 51-64, 2006.

10. Amaravadi RK, Yu D, Lum JJ, et al: Autophagy inhibition enhances therapy-induced apoptosis in a Myc-induced model of lymphoma. J Clin Invest 117: 326-336, 2007.

11. Rosenfeldt MT and Ryan KM: The role of autophagy in tumour development and cancer therapy. Expert Rev Mol Med 11: e36, 2009.

12. Jin S and White E: Tumor suppression by autophagy through the management of metabolic stress. Autophagy 4: 563-566, 2008.

13. O'Donovan TR, O'Sullivan GC and McKenna SL: Induction of autophagy by drug-resistant esophageal cancer cells promotes their survival and recovery following treatment with chemotherapeutics. Autophagy 7: 509-524, 2011.

14. Sun WL, Chen J, Wang YP and Zheng H: Autophagy protects breast cancer cells from epirubicin-induced apoptosis and facilitates epirubicin-resistance development. Autophagy 7 : 1035-1044, 2011

15. Seibenhener ML, Geetha T and Wooten MW: Sequestosome 1/ p62 - more than just a scaffold. FEBS Lett 581: 175-179, 2007.

16. Komatsu M, Kurokawa H, Waguri S, et al: The selective autophagy substrate p62 activates the stress responsive transcription factor Nrf2 through inactivation of Keap1. Nat Cell Biol 12: 213-223, 2010.
17. Bjorkoy G, Lamark T and Johansen T: p62/SQSTM1: a missing link between protein aggregates and the autophagy machinery. Autophagy 2: 138-139, 2006

18. Kirkin V, McEwan DG, Novak I and Dikic I: A role for ubiquitin in selective autophagy. Mol Cell 34: 259-269, 2009.

19. Shvets E, Fass E, Scherz-Shouval R and Elazar Z: The N-terminus and Phe52 residue of LC3 recruit p62/SQSTM1 into autophagosomes. J Cell Sci 121: 2685-2695, 2008.

20. Linares JF, Amanchy R, Greis K, Diaz-Meco MT and Moscat J: Phosphorylation of p62 by cdk1 controls the timely transit of cells through mitosis and tumor cell proliferation. Mol Cell Biol 31: 105-117, 2011.

21. Kabeya Y, Mizushima N, Ueno T, et al: LC3, a mammalian homologue of yeast Apg8p, is localized in autophagosome membranes after processing. EMBO J 19: 5720-5728, 2000.

22. Bjorkoy G, Lamark T, Brech A, et al: p62/SQSTM1 forms protein aggregates degraded by autophagy and has a protective effect on huntingtin-induced cell death. J Cell Biol 171: 603-614, 2005.

23. Solomon VR and Lee H: Chloroquine and its analogs: a new promise of an old drug for effective and safe cancer therapies. Eur J Pharmacol 625: 220-233, 2009.

24. Tanida I, Minematsu-Ikeguchi N, Ueno T and Kominami E: Lysosomal turnover, but not a cellular level, of endogenous LC3 is a marker for autophagy. Autophagy 1: 84-91, 2005.

25. Kaini RR and Hu CA: Synergistic killing effect of chloroquine and androgen deprivation in LNCaP cells. Biochem Biophys Res Commun 425: 150-156, 2012.

26. Sasaki K, Tsuno NH, Sunami E, et al: Chloroquine potentiates the anti-cancer effect of 5-fluorouracil on colon cancer cells. BMC Cancer 10: 370, 2010 